\section{Conhecimento e percepção sobre exercício físico em uma população adulta urbana do sul do Brasil}

\author{
Knowledge and perceptions of physical exercise \\ in an adult urban population in Southern Brazil
}

Marlos Rodrigues Domingues 1

Cora Luiza Pavin Araújo 1

Denise Petrucci Gigante 1

\section{Introdução}

1 Programa de Pós-graduação em Epidemiologia, Departamento de Medicina Social, Faculdade de Medicina, Universidade Federal de Pelotas, Pelotas, Brasil.

Correspondência Marlos Rodrigues Domingues Programa de Pós-graduação em Epidemiologia, Departamento de Medicina Social, Faculdade de Medicina, Universidade Federal de Pelotas. Rua Pedro Armando Gatti 158, Rio Grande, RS 96216-080, Brasil. coriolis@vetorial.net

\section{Abstract}

This study evaluates perception and knowledge of physical exercise in an urban adult population and relates knowledge level to behavioral, demographic, and socioeconomic factors, using a cross-sectional, populationbased approach with 3,182 participants aged 20 or older. The questionnaire originated a score (range 0-25 points). Mean score for the sample was 17.1 ( $S D=4.0)$. After adjustment, female gender, age 30 to 40 years, higher social strata, and schooling were strongly associated ( $p<0.001$ ) with the outcome (knowledge). Weaker associations ( $p \leq 0.01)$ were observed between the score and higher body mass index, level of physical activity, and white skin color. Based on the results, specific benefits from exercising are still unknown to some population groups. Counseling by teachers on the advantages of an active lifestyle is still not common practice.

Physical Exercises; Adult Health; Urban Population
O sedentarismo é visto atualmente como um problema mundial de saúde 1,2. Entre as razões que levam à inatividade, um dos possíveis fatores é o desconhecimento sobre como se exercitar, as finalidades de cada exercício, limitações de alguns grupos populacionais e percepções distorcidas em relação aos benefícios do movimento.

Freqüentemente considerados como equivalentes, os termos "atividade física" e "exercício físico” não são sinônimos. Segundo Caspersen et al. 3, atividade física é qualquer movimento corporal produzido pela musculatura esquelética, que resulte em um gasto energético maior do que os níveis de repouso, enquanto que exercício físico é toda atividade física planejada, estruturada e repetitiva que tem por objetivo a melhoria e a manutenção da aptidão física.

O fato de possuir o conhecimento não implica necessariamente a prática de exercício físico, mas sem o conhecimento e percepção corretos sobre o tema é improvável que atitudes sejam tomadas no sentido de alterar um padrão comportamental. Além disso, maiores níveis de conhecimento sobre exercício físico podem fazer com que os níveis de sedentarismo não aumentem 4 .

Sabe-se hoje que o exercício físico pode ser um fator protetor para uma série de males, en- 
tre os quais destacam-se: obesidade, doenças cardiovasculares, diabetes, osteoporose, depressão e maior morbi-mortalidade por qualquer causa 5,6. No entanto, muito desse conhecimento não é adequadamente divulgado fora do meio acadêmico, permanecendo oculto para grande parte da população. Os motivos que levam ao desconhecimento vão da falta de vontade própria em buscar informação até a inexistência de programas governamentais de esclarecimento, passando pelos profissionais de saúde que, muitas vezes, também ignoram o valor do exercício físico e/ou não são efetivos no incentivo à prática regular de exercícios físicos.

Com a finalidade de avaliar o conhecimento e a percepção sobre exercício físico em uma população adulta urbana, foi feito um estudo procurando medir por meio de um escore edescrever as características dos indivíduos com relação ao assunto, levando em conta nesta análise as características sócio-econômicas, demográficas e comportamentais da população.

\section{Material e métodos}

Foi realizado um estudo transversal, de base populacional, de março a maio de 2002, em sistema de consórcio entre o grupo de mestrandos do Programa de Pós-graduação em Epidemiologia da Universidade Federal de Pelotas. O consórcio é a união de vários pesquisadores na elaboração de um questionário que é aplicado à população com questões de interesse geral e questões específicas de cada pesquisador.

A amostra, representativa da população de Pelotas, Rio Grande do Sul, foi selecionada por conglomerados em dois estágios e composta por pessoas de ambos os sexos com idade igual ou superior a vinte anos. Os 281 setores censitários da zona urbana do município foram divididos de acordo com quatro estratos de escolaridade do chefe da família, e oitenta setores foram sorteados.

De posse da listagem de domicílios elegíveis (excluindo os desabitados ou puramente comerciais), foram sorteados sistematicamente vinte domicílios de cada setor para compor a amostra, chegando-se a um total de 1.600 domicílios e uma previsão inicial de 3.360 pessoas elegíveis para a amostra.

Cálculos amostrais posteriores (por diferenças de médias) demonstraram que a amostra obtida permitiu um poder estatístico igual ou superior a $80 \%$ para todas as variáveis, com exceção do Índice de Massa Corporal (IMC), que exigiria uma amostra muito superior, inviabilizando o estudo.

Após ampla revisão bibliográfica e contato com pesquisadores da área não foi possível identificar um instrumento validado para avaliar conhecimento sobre exercício físico. Sendo assim, elaborou-se um questionário que foi testado em três situações de campo antes de ser definitivamente aplicado. Esses testes serviram para aperfeiçoar o instrumento, tornando-o mais adequado à compreensão da população estudada.

O objetivo deste questionário era identificar o conhecimento sobre: benefícios do exercício físico, prejuízos do sedentarismo, limitações e finalidades do exercício físico, e ainda avaliar a percepção sobre o assunto.

$\mathrm{Na}$ Tabela 1 podem ser encontradas as nove questões fechadas, de simples e múltipla escolha, com a respectiva pontuação para elaboração do escore. As alternativas em destaque indicam as respostas consideradas corretas. A primeira e a última questão não faziam parte do escore, apenas da análise de percepção.

As questões possuíam pesos diferenciados. Esta ponderação foi necessária uma vez que algumas apresentavam maior grau de dificuldade e/ou tratavam de assuntos de maior relevância. O grau de dificuldade de algumas questões pôde ser percebido durante os estudos-piloto. Todos os valores das questões foram estabelecidos anteriormente à coleta de dados final e elaboração do escore.

Com base nos dados do questionário foi possível gerar dois conjuntos de informação um escore de conhecimento e uma descrição dos itens pesquisados. O escore foi obtido por meio das respostas e a pontuação total poderia variar entre 0 (zero) e 25 (vinte e cinco). Respostas erradas não diminuíam a pontuação, apenas não acrescentavam pontos ao escore.

Para o escore (criado para avaliar o conhecimento populacional) foi calculado o coeficiente Alfa de Cronbach, que ficou em 0,83. As correlações entre as variáveis componentes do escore e entre as variáveis e o escore ficaram na maioria entre 0,15 e 0,35 (valores extremos indo de 0,05 a 0,60 ), mostrando que nenhuma variável teve um peso determinante no escore final e que todas tiveram importância semelhante para a formação do mesmo.

Para o trabalho de campo, uma equipe de quarenta entrevistadoras foi utilizada após seleção, treinamento e testagem para a coleta dos 
Tabela 1

Questionário específico para avaliar a percepção e o conhecimento da população sobre exercício físico (com valores atribuídos para elaboração do escore).

Pelotas, Rio Grande do Sul, Brasil, 2002.

Como o(a) Sr(a). considera seu conhecimento sobre exercícios físicos?

(Ler os itens e escolher apenas um)

() sabe o suficiente

() gostaria de aprender mais

( ) não acha necessário saber essas coisas

( ) não tem nenhum conhecimento

( ) IGN

Para que uma pessoa cresça e envelheça com uma boa saúde, o(a) $\mathrm{Sr}(a)$. considera o exercício físico: (Ler os itens e escolher apenas um)

(0) sem importância

(0) pouco importante

(1) muito importante

2) indispensável

(0) IGN

Das seguintes doenças, quais o(a) $\mathrm{Sr}(\mathrm{a})$. acha que PODERIAM ser prevenidas com o hábito de fazer exercício físico? (Ler itens)

$\begin{array}{llll}\text { Pressão alta } & (0) \text { não } & \text { (1) sim } & \text { (0) IGN } \\ \text { Câncer de pele } & (1) \text { não } & \text { (0) sim } & \text { (0) IGN } \\ \text { Osteoporose (ossos fracos) } & \text { (0) não } & \text { (1) sim } & \text { (0) IGN } \\ \text { Colesterol alto } & \text { (0) não } & \text { (1) sim } & \text { (0) IGN }\end{array}$

Quais destas pessoas o(a) $\mathrm{Sr}(a)$. acha que PODERIAM fazer exercícios físicos? (Ler itens)

$\begin{array}{llll}\text { Uma mulher no início da gravidez } & (0) \text { não } & \text { (1) sim } & \text { (0) IGN } \\ \begin{array}{l}\text { Alguém com osteoporose e problemas } \\ \text { no coração }\end{array} & \text { (0) não } & \text { (2) sim } & \text { (0) IGN } \\ \text { Um idoso com mais de } 90 \text { anos } & \text { (0) não } & \text { (1) sim } & \text { (0) IGN } \\ \text { Uma criança com menos de } 10 \text { anos } & \text { (0) não } & \text { (1) sim } & \text { (0) IGN }\end{array}$

Destes exemplos, qual seria o tempo MíNIMO para melhorar sua saúde com exercícios físicos? (Ler itens e escolher apenas um)

(0) 10 minutos, 4 vezes por semana

(0) 2 horas por dia, todos os dias

(2) 30 minutos, 3 vezes por semana

(0) 1 hora, 1 vez por semana

(0) IGN

A falta de exercício físico PODE fazer com que a pessoa tenha: (Ler itens)
$\begin{array}{llll}\text { Diabetes (açúcar no sangue) } & \text { (0) não } & \text { (2) } \operatorname{sim} & \text { (0) IGN } \\ \text { Diarréia } & \text { (1) não } & \text { (0) } \operatorname{sim} & \text { (0) IGN } \\ \text { Problemas de circulação } & \text { (0) não } & \text { (1) } \operatorname{sim} & \text { (0) IGN } \\ \text { Meningite } & \text { (1) não } & \text { (0) } \operatorname{sim} & \text { (0) IGN }\end{array}$

Quais destes problemas do dia-a-dia o(a) Sr(a). acha que o exercício físico pode ajudar a combater? (Ler itens)

$\begin{array}{llll}\text { Estresse } & (0) \text { não } & \text { (1) } \operatorname{sim} & \text { (0) IGN } \\ \text { Insônia (dificuldade para dormir) } & (0) \text { não } & \text { (1) sim } & \text { (0) IGN } \\ \text { Ansiedade (nervosismo) } & (0) \text { não } & \text { (1) sim } & \text { (0) IGN } \\ \text { Depressão } & \text { (0) não } & \text { (1) sim } & \text { (0) IGN }\end{array}$

(continua) dados do consórcio. Os entrevistados consentiam verbalmente em participar da pesquisa antes do início da entrevista.

As variáveis independentes estudadas foram definidas da seguinte forma: Sexo - masculino ou feminino; Idade - coletada em anos completos; Cor da pele - observada pela entrevistadora e classificada como branca ou nãobranca; Nivel social - dados coletados e classificados com base no questionário padronizado da Associação Nacional de Empresas de Pesquisa 7: A, B, C, D ou E; IMC - baseado no peso e altura referidos pelo entrevistado. A classificação nutricional utilizada foi aquela recomendada pela Organização Mundial da Saúde 8: desnutrido/normal (abaixo de 25,0 ), sobrepeso (entre 25,0 e 30,0 ) e obesidade (acima de 30,0; Prática de religião - declaração do entrevistado como praticante ou não praticante; $E s$ colaridade - coletada em anos completos de estudo; Autopercepção de saúde - opinião do entrevistado: Excelente, Muito boa, Boa, Regular ou Ruim; Situação conjugal - sem companheiro (solteiro, separado, desquitado ou viúvo) ou com companheiro (namorado, marido/ esposa ou companheiro); Tabagismo - fumante ( 1 ou + cigarros/dia, há mais de 1 mês), nãofumante (nunca fumou) e ex-fumante; e Atividade física - classificados como suficientemente ativos (atividade semanal $\geq 150$ minutos) ou insuficientemente ativos (atividade semanal < 150 minutos), de acordo com a versão curta do International Physical Activity Questionnaire 9.

Todos os questionários foram inicialmente revisados pela própria entrevistadora e, a seguir, pelo seu supervisor. Os dados foram digitados duas vezes (Epi Info 6.04b) a fim de identificar erros de digitação. A análise de consistência e a análise estatística foram realizadas utilizando-se o pacote estatístico Stata 7.0.

Os supervisores de trabalho de campo realizaram uma revisita em $10 \%$ da amostra utilizando uma versão resumida do questionário. Estes questionários de controle de qualidade foram usados para cálculo de concordância entre observadores (coeficiente Kappa).

Inicialmente foi feita a descrição da amostra e em seguida realizou-se uma análise bruta da variável desfecho (escore de conhecimento) com as variáveis independentes. Devido às grandes diferenças apresentadas nos resultados entre homens e mulheres, toda análise (descritiva, bruta e ajustada) foi realizada para toda a amostra e separadamente para cada sexo. Em todas as análises foi levado em conta o efeito do desenho amostral para o desfecho (DEF $=3,6$ ).

Para a realização da análise bivariada, o teste usado foi o de Wald, e o escore foi categori- 
zado em quartis $(0-14 ; 15-17 ; 18-20 ; 21-25)$. Os quartis permaneceram em forma de valor $a b-$ soluto, uma vez que até o momento não existe referencial teórico amplamente aceito para categorizar o conhecimento.

Nesta fase (análise bruta), as variáveis que apresentaram valor de $\mathrm{p}<0,2$ foram mantidas e levadas para análise ajustada, enquanto que as demais foram descartadas. Para as variáveis com mais de duas categorias foi realizado teste para verificar a tendência linear, e para as de duas categorias o teste de heterogeneidade de médias.

Durante a análise ajustada (regressão linear múltipla) o escore foi tratado de forma contínua (0-25). Foi realizado diagnóstico do modelo por meio de gráficos de resíduos e de resíduos padronizados com os valores preditos.

\section{Resultados}

Ao final da pesquisa, excluindo-se 5,6\% de perdas e recusas, obteve-se uma amostra total de 3.182 pessoas.

O escore de conhecimento em toda população apresentou o valor médio de 17,1 (desvio padrão $=4,0)$. Entre os homens, o valor foi de 16,7 $(\mathrm{DP}=3,9)$ e entre as mulheres $17,5(\mathrm{DP}=4,1)$.

A Tabela 2 descreve as freqüências das variáveis referentes à percepção do exercício físico para toda a amostra e, separadamente para homens e mulheres. Pode ser observado que os homens referem saber mais em relação às mulheres, no entanto, elas responderam mais corretamente às questões em geral, e ambos os sexos referem que gostariam de saber mais sobre $\mathrm{o}$ assunto.

A grande maioria da população reconhece a importância do exercício físico, porém menos de $20 \%$ considera-o como sendo indispensável nos processos de crescimento e envelhecimento saudáveis.

Mais da metade da população identifica que realizar exercício físico por trinta minutos, três vezes por semana, é o mínimo necessário para que os benefícios sobre a saúde possam ser percebidos. Além disso, mais de $50 \%$ da amostra consideram a caminhada como um excelente exercício para o emagrecimento.

Ainda nesta tabela pode ser observado algum desconhecimento, pois persiste um certo preconceito quanto à possibilidade da prática de exercício físico por populações com aparentes limitações, como pessoas idosas e/ou com certos problemas de saúde.

Chama a atenção que entre os cinco problemas mais citados como alteráveis pelo exer-

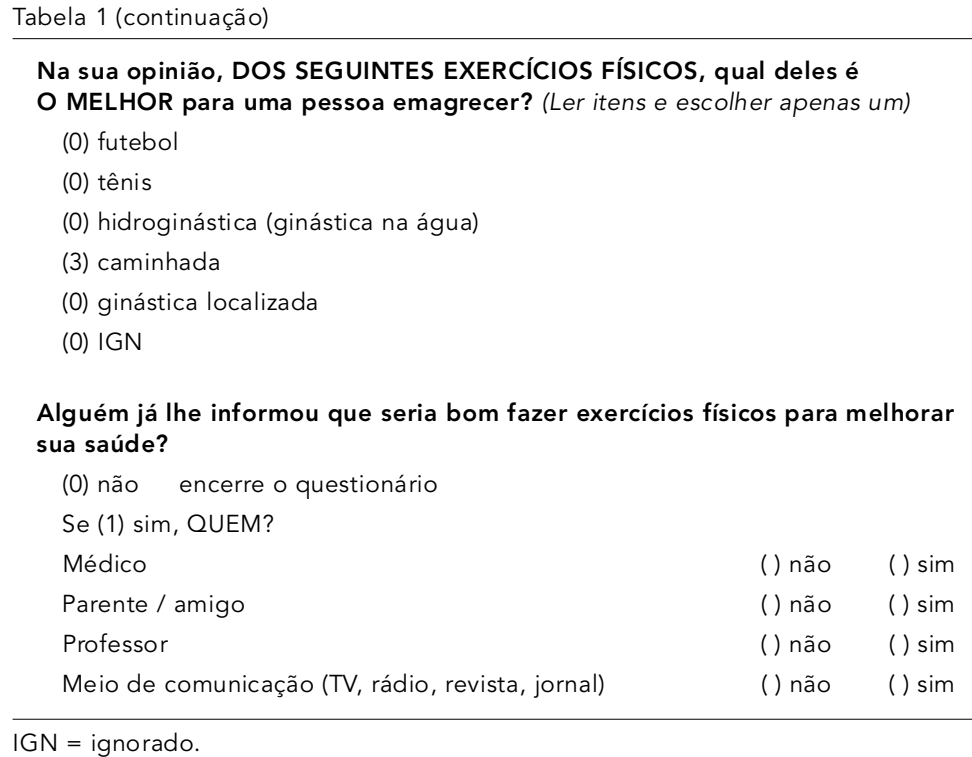

cício físico, quatro foram mais relacionados a aspectos emocionais: estresse, depressão, ansiedade e insônia. Além destes quatro, os problemas circulatórios ficaram em segundo lugar. Outros problemas como colesterol alto, diabetes, hipertensão e osteoporose foram menos citados.

Os meios de comunicação foram a fonte de informação sobre os benefícios do exercício físico mais freqüente, seguida pelo médico e por parente ou amigo. Cabe salientar que mais de um quinto da amostra refere que nunca recebeu este tipo de informação.

Na Tabela 3 pode-se observar a média e o desvio padrão do escore de conhecimento sobre exercício físico, segundo as diversas variáveis independentes, incluídas neste estudo. Além disso, são apresentados também os valores dos escores categorizados por quartis de freqüência.

Considerando-se a amostra toda, foram significativas as associações entre as categorias do escore e sexo, idade, cor da pele, nível social, escolaridade, tabagismo, IMC e autopercepção de saúde.

A Tabela 4 apresenta os resultados médios do escore por categoria e as associações entre as categorias do escore e as variáveis independentes, separadamente para homens e mulheres. Deve-se destacar que as associações observadas mostraram-se diferentes entre os sexos. Para as mulheres todas as associações, exceto IMC e tabagismo, apresentaram significância estatística. Já para os homens, apenas o nível social, a escolaridade, o tabagismo e o IMC estiveram associados. Em relação à classificação quanto ao nível de atividade física, os homens 
Distribuição das variáveis referentes à percepção e conhecimento do exercício físico para toda a amostra e, separadamente, para homens e mulheres $(n=3.182)$. Pelotas, Rio Grande do Sul, Brasil, 2002.

\begin{tabular}{|c|c|c|c|}
\hline \multirow[t]{2}{*}{ Variável } & \multicolumn{3}{|c|}{ Prevalências (\%) } \\
\hline & Todos & Homens & Mulheres \\
\hline \multicolumn{4}{|l|}{ Autopercepção do conhecimento sobre exercício físico } \\
\hline Sabe o suficiente & 20,6 & 25,9 & 16,6 \\
\hline Gostaria de aprender mais & 60,8 & 57,6 & 63,2 \\
\hline Não acha necessário saber essas coisas & 5,6 & 5,1 & 6,0 \\
\hline Não tem nenhum conhecimento & 12,0 & 10,7 & 13,0 \\
\hline IGN & 1,0 & 0,7 & 1,2 \\
\hline \multicolumn{4}{|l|}{ Importância do exercício no crescimento/envelhecimento } \\
\hline Sem importância & 0,6 & 0,7 & 0,6 \\
\hline Pouco importante & 2,1 & 2,0 & 2,1 \\
\hline Muito importante & 77,2 & 74,8 & 79,0 \\
\hline Indispensável & 19,2 & 21,8 & 17,3 \\
\hline IGN & 0,9 & 0,7 & 1,0 \\
\hline \multicolumn{4}{|l|}{ Freqüência mínima de exercício para obtenção de saúde } \\
\hline 10 minutos, 4 vezes por semana & 10,9 & 11,4 & 10,4 \\
\hline 2 horas por dia, todos os dias & 16,9 & 20,0 & 14,6 \\
\hline 30 minutos, 3 vezes por semana & 57,7 & 55,9 & 59,1 \\
\hline 1 hora, 1 vez por semana & 11,7 & 10,4 & 17,7 \\
\hline IGN & 2,8 & 2,3 & 3,2 \\
\hline \multicolumn{4}{|l|}{ Melhor exercício para o emagrecimento } \\
\hline Futebol & 12,0 & 20,8 & 5,4 \\
\hline Tênis & 1,6 & 2,1 & 1,1 \\
\hline Hidroginástica & 13,8 & 15,1 & 12,9 \\
\hline Caminhada & 54,4 & 46,3 & 60,6 \\
\hline Ginástica localizada & 16,4 & 14,5 & 17,8 \\
\hline IGN & 1,8 & 1,2 & 2,2 \\
\hline \multicolumn{4}{|l|}{ Quem pode executar exercício físico } \\
\hline Uma criança com menos de 10 anos & 84,8 & 83,2 & 85,9 \\
\hline Uma mulher no início da gravidez & 81,0 & 81,4 & 80,7 \\
\hline Um idoso com mais de 90 anos & 66,4 & 67,4 & 65,7 \\
\hline Uma pessoa com osteoporose e problemas cardíacos & 59,8 & 56,2 & 62,6 \\
\hline \multicolumn{4}{|l|}{ Identificação do exercício como protetor contra doenças } \\
\hline Estresse & 93,6 & 93,3 & 93,8 \\
\hline Depressão & 87,7 & 87,0 & 88,2 \\
\hline Ansiedade & 87,3 & 86,1 & 88,3 \\
\hline Insônia & 81,6 & 80,7 & 82,3 \\
\hline Colesterol alto & 75,4 & 73,6 & 76,7 \\
\hline Hipertensão & 71,9 & 70,9 & 72,7 \\
\hline Câncer de pele & 17,1 & 19,8 & 15,0 \\
\hline Osteoporose & 71,0 & 66,4 & 74,4 \\
\hline \multicolumn{4}{|l|}{ Identificação do sedentarismo como fator de risco } \\
\hline Problemas de circulação & 91,8 & 91,3 & 92,1 \\
\hline Diarréia & 10,5 & 13,5 & 8,2 \\
\hline Meningite & 11,7 & 13,7 & 10,2 \\
\hline Diabetes & 42,8 & 42,4 & 43,0 \\
\hline \multicolumn{4}{|l|}{ Fonte de informação sobre benefícios da atividade física } \\
\hline Meio de comunicação & 51,2 & 49,3 & 52,6 \\
\hline Médico & 51,1 & 44,6 & 56,0 \\
\hline Parente ou amigo & 49,7 & 48,4 & 50,7 \\
\hline Professor & 19,1 & 24,0 & 15,3 \\
\hline Nunca recebeu informação & 21,0 & 23,6 & 18,8 \\
\hline
\end{tabular}

IGN = ignorado. 
Distribuição das variáveis demográficas, socioeconômicas, comportamentais, antropométricas e sobre a autopercepção de saúde na amostra total, segundo as categorias (quartis) de escores de conhecimento sobre exercício ( $n=3.182$ ). Pelotas, Rio Grande do Sul, Brasil, 2002.

\begin{tabular}{|c|c|c|c|c|c|c|c|}
\hline Variável & $\%$ & $X( \pm D P)$ & $\begin{array}{c}0-14 \\
(\%)\end{array}$ & $\begin{array}{c}15-17 \\
(\%)\end{array}$ & $\begin{array}{c}18-20 \\
(\%)\end{array}$ & $\begin{array}{c}21-25 \\
(\%)\end{array}$ & $p$ \\
\hline Sexo & & & & & & & $<0,0011$ \\
\hline Masculino & 43,2 & $16,7(3,9)$ & 26,1 & 29,2 & 29,2 & 15,5 & \\
\hline Feminino & 56,8 & $17,5(4,1)$ & 19,5 & 26,4 & 29,6 & 24,5 & \\
\hline Idade & & & & & & & $<0,0012$ \\
\hline 20-29 & 22,6 & $17,3(3,7)$ & 22,4 & 26,1 & 31,6 & 19,9 & \\
\hline $30-39$ & 21,4 & $17,5(3,5)$ & 18,1 & 30,6 & 29,8 & 21,5 & \\
\hline $40-49$ & 20,9 & $17,5(3,8)$ & 20,5 & 26,7 & 28,8 & 24,0 & \\
\hline $50-59$ & 16,7 & $17,6(3,9)$ & 19,7 & 25,7 & 30,8 & 23,8 & \\
\hline $60-69$ & 9,6 & $16,6(4,0)$ & 24,7 & 31,3 & 28,7 & 15,3 & \\
\hline$\geq 70$ & 8,7 & $14,7(5,5)$ & 39,5 & 25,7 & 22,8 & 12,0 & \\
\hline Cor da pele & & & & & & & 0,0041 \\
\hline Branca & 84,7 & $17,2(4,1)$ & 22,2 & 26,4 & 29,7 & 21,7 & \\
\hline Negra/parda & 15,3 & $16,7(3,8)$ & 23,2 & 34,2 & 28,0 & 14,6 & \\
\hline Nível social & & & & & & & $<0,0012$ \\
\hline Nível A & 4,6 & $19,3(3,5)$ & 7,5 & 17,7 & 32,6 & 42,2 & \\
\hline Nível B & 18,9 & $18,5(3,8)$ & 11,2 & 21,2 & 36,8 & 30,8 & \\
\hline Nível C & 40,0 & $17,4(3,9)$ & 19,9 & 27,7 & 30,3 & 22,1 & \\
\hline Nível D & 32,4 & $16,0(4,0)$ & 31,6 & 31,5 & 21,5 & 11,4 & \\
\hline Nível E & 4,0 & $14,8(4,1)$ & 41,0 & 36,2 & 16,5 & 6,3 & \\
\hline Escolaridade & & & & & & & $<0,0012$ \\
\hline 0 & 7,0 & $14,1(4,7)$ & 48,9 & 27,4 & 17,9 & 5,8 & \\
\hline $1-4$ & 20,6 & $15,9(4,0)$ & 31,7 & 34,8 & 22,4 & 11,1 & \\
\hline $5-8$ & 33,6 & $16,9(3,9)$ & 24,6 & 28,4 & 30,3 & 16,7 & \\
\hline $9-11$ & 24,5 & $18,2(3,3)$ & 13,2 & 25,9 & 34,4 & 26,5 & \\
\hline$\geq 12$ & 14,2 & $19,5(3,1)$ & 5,8 & 18,4 & 35,0 & 40,8 & \\
\hline Tabagismo & & & & & & & 0,00042 \\
\hline Não-fumante & 52,4 & $17,4(4,1)$ & 20,2 & 27,0 & 29,4 & 23,4 & \\
\hline Ex-fumante & 19,7 & $17,1(4,0)$ & 24,2 & 25,0 & 31,3 & 19,5 & \\
\hline Fumante & 27,9 & $16,8(3,8)$ & 25,0 & 30,6 & 28,2 & 16,2 & \\
\hline Situação conjugal & & & & & & & 0,221 \\
\hline Com companheiro & 61,3 & $17,2(3,9)$ & 21,5 & 28,6 & 28,8 & 21,1 & \\
\hline Sem companheiro & 38,7 & $17,0(4,3)$ & 23,7 & 25,9 & 30,6 & 19,8 & \\
\hline Prática de religião & & & & & & & 0,331 \\
\hline Sim & 54,0 & $17,1(4,2)$ & 22,7 & 27,8 & 28,1 & 21,4 & \\
\hline Não & 46,0 & $17,2(3,9)$ & 21,9 & 27,4 & 31,0 & 19,7 & \\
\hline IMC & & & & & & & 0,032 \\
\hline Desnutrição/Normal $(<25,0)$ & 50,6 & $17,2(3,8)$ & 21,7 & 28,7 & 30,1 & 19,5 & \\
\hline Sobrepeso $(25,0-29,9)$ & 35,0 & $17,3(4,0)$ & 22,5 & 25,0 & 30,9 & 21,6 & \\
\hline Obesidade $(\geq 30,0)$ & 14,4 & $17,1(4,1)$ & 17,8 & 27,4 & 28,3 & 26,5 & \\
\hline Atividade física & & & & & & & 0,131 \\
\hline Suficientemente ativo & 58,9 & $17,4(3,7)$ & 20,4 & 27,4 & 30,8 & 21,4 & \\
\hline Insuficientemente ativo & 41,1 & $17,0(4,1)$ & 23,8 & 28,2 & 28,2 & 19,8 & \\
\hline Autopercepção de saúde & & & & & & & $<0,0012$ \\
\hline Excelente & 3,9 & $15,5(4,1)$ & 34,4 & 35,2 & 19,2 & 11,2 & \\
\hline Muito boa & 24,2 & $16,9(3,8)$ & 23,5 & 30,2 & 30,0 & 16,3 & \\
\hline Boa & 47,2 & $17,2(3,9)$ & 22,2 & 27,3 & 29,6 & 28,9 & \\
\hline Regular & 15,2 & $18,0(3,7)$ & 16,5 & 26,2 & 29,6 & 27,7 & \\
\hline Ruim & 9,5 & $17,6(3,9)$ & 20,7 & 22,3 & 33,0 & 24,0 & \\
\hline
\end{tabular}

Nota: O número máximo de não-respondentes foi de 135 (4,2\%) para a variável IMC.

1 Teste de Wald para heterogeneidade de médias. 2 Teste de Wald para tendência linear. 
Distribuição das variáveis demográficas, socioeconômicas, comportamentais, antropométricas e sobre a autopercepção de saúde estratificada por sexo, segundo os valores médios de escore de conhecimento sobre exercício. Pelotas, Rio Grande do Sul, Brasil, 2002.

\begin{tabular}{|c|c|c|c|c|c|c|}
\hline \multirow[t]{2}{*}{ Variável } & \multicolumn{3}{|c|}{ Mulheres $(n=1.808)$} & \multicolumn{3}{|c|}{ Homens ( $n=1.374$ ) } \\
\hline & $\%$ & $X( \pm D P)$ & $P$ & $\%$ & $X( \pm D P)$ & $p$ \\
\hline Idade & & & $<0,0011$ & & & 0,31 \\
\hline $20-29$ & 21,3 & $17,9(3,7)$ & & 24,3 & $16,6(3,5)$ & \\
\hline $30-39$ & 20,3 & $18,2(3,3)$ & & 22,8 & $16,7(3,7)$ & \\
\hline $40-49$ & 20,8 & $17,8(4,0)$ & & 21,2 & $17,2(3,6)$ & \\
\hline $50-59$ & 17,7 & $18,0(3,8)$ & & 15,5 & $17,0(3,8)$ & \\
\hline $60-69$ & 10,1 & $16,9(4,1)$ & & 9,0 & $16,3(4,0)$ & \\
\hline$\geq 70$ & 9,8 & $14,3(5,5)$ & & 7,2 & $15,4(5,6)$ & \\
\hline Cor da pele & & & 0,012 & & & 0,112 \\
\hline Branca & 84,4 & $17,6(4,1)$ & & 81,1 & $16,7(3,9)$ & \\
\hline Negra/Parda & 15,6 & $16,9(3,9)$ & & 14,9 & $16,4(3,5)$ & \\
\hline Nível social & & & $<0,0011$ & & & $<0,0011$ \\
\hline Nível A & 4,1 & $19,7(3,7)$ & & 5,3 & $18,9(3,1)$ & \\
\hline Nível B & 18,7 & $18,9(3,9)$ & & 19,3 & $17,9(3,5)$ & \\
\hline Nível C & 40,3 & $17,8(3,7)$ & & 39,8 & $16,9(3,9)$ & \\
\hline Nível D & 32,7 & $16,3(4,1)$ & & 31,9 & $15,6(3,8)$ & \\
\hline Nível E & 4,2 & $15,0(4,7)$ & & 3,7 & $14,4(2,9)$ & \\
\hline Escolaridade & & & $<0,0011$ & & & $<0,0011$ \\
\hline 0 & 8,5 & $14,1(5,0)$ & & 5,0 & $14,2(4,1)$ & \\
\hline $1-4$ & 21,2 & $16,1(4,1)$ & & 19,9 & $15,6(3,9)$ & \\
\hline $5-8$ & 31,2 & $17,5(3,7)$ & & 36,8 & $16,1(3,9)$ & \\
\hline $9-11$ & 25,0 & $18,6(3,3)$ & & 23,9 & $17,6(3,3)$ & \\
\hline$\geq 12$ & 14,1 & $19,8(3,2)$ & & 14,4 & $19,0(3,1)$ & \\
\hline Tabagismo & & & 0,071 & & & 0,0031 \\
\hline Não-fumante & 59,6 & $17,5(4,3)$ & & 42,9 & $17,1(3,8)$ & \\
\hline Ex-fumante & 16,2 & $17,8(3,7)$ & & 24,3 & $16,5(4,1)$ & \\
\hline Fumante & 24,2 & $17,3(3,8)$ & & 32,8 & $16,3(3,8)$ & \\
\hline Situação conjugal & & & 0,022 & & & 0,632 \\
\hline Com companheiro & 55,2 & $17,7(3,7)$ & & 69,3 & $16,7(3,9)$ & \\
\hline Sem companheiro & 44,8 & $17,3(4,5)$ & & 30,7 & $16,5(3,8)$ & \\
\hline Prática de religião & & & 0,012 & & & 0,732 \\
\hline Sim & 59,7 & $17,4(4,2)$ & & 46,6 & $16,5(4,1)$ & \\
\hline Não & 40,3 & $17,6(4,0)$ & & 53,4 & $16,8(3,7)$ & \\
\hline IMC & & & 0,611 & & & 0,0021 \\
\hline Desnutrição/Normal $(<25,0)$ & 53,8 & $17,7(3,8)$ & & 46,7 & $16,4(3,7)$ & \\
\hline Sobrepeso $(25,0-29,9)$ & 30,9 & $17,6(4,1)$ & & 40,1 & $16,9(4,0)$ & \\
\hline Obesidade $(\geq 30,0)$ & 15,3 & $17,9(4,1)$ & & 13,2 & $17,3(3,9)$ & \\
\hline Atividade física & & & 0,0012 & & & 0,942 \\
\hline Suficientemente ativo & 58,2 & $17,9(3,7)$ & & 59,8 & $16,8(3,6)$ & \\
\hline Insuficientemente ativo & 41,8 & $17,2(4,2)$ & & 40,2 & $16,6(4,0)$ & \\
\hline Autopercepção de saúde & & & $<0,0011$ & & & 0,481 \\
\hline Excelente & 5,6 & $15,5(4,2)$ & & 1,7 & $15,3(3,7)$ & \\
\hline Muito boa & 28,3 & $17,1(3,8)$ & & 18,8 & $16,5(3,8)$ & \\
\hline Boa & 44,4 & $17,7(3,9)$ & & 50,9 & $16,7(3,7)$ & \\
\hline Regular & 14,2 & $18,7(3,7)$ & & 16,4 & $17,3(3,7)$ & \\
\hline Ruim & 7,5 & $18,7(3,4)$ & & 12,1 & $16,7(4,0)$ & \\
\hline
\end{tabular}

1 Teste de Wald para tendência linear.

2 Teste de Wald para heterogeneidade de médias. 
apresentaram níveis ligeiramente mais altos de atividade, embora esta diferença não seja estatisticamente significativa.

O diagnóstico por meio dos gráficos de resíduos (normal e padronizado) mostrou que o modelo estava adequado, sem fuga da linearidade, apresentando normalidade e homogeneidade de variância.

A Tabela 5 apresenta os resultados da regressão linear múltipla das variáveis independentes sobre o conhecimento para a amostra total e estratificada por sexo. Conforme a apresentação na tabela, as variáveis eram ajustadas em relação às anteriores. Observando-se a Tabela 5 , no que se refere à amostra total, apresentaram significância estatística o sexo, a idade, a cor da pele, o nível social, a escolaridade, o nível de atividade física e o IMC. Os coeficientes $\beta$ indicam a magnitude da modificação nos escores de conhecimento, em cada categoria das variáveis independentes, usando como base a categoria com os piores escores. Entre os homens, a associação com o desfecho ( $\mathrm{p}<$ 0,05 ) ocorreu apenas para nível social, escolaridade e IMC. Entre as mulheres, o escore de conhecimento mostrou-se significativamente associado com idade, nível social, escolaridade, atividade física, cor da pele e situação conjugal.

O teste Kappa para a questão escolhida (fonte de informação sobre exercício físico) mostrou um coeficiente de concordância igual a 0,4 , que corresponde a um nível moderado. Este teste não mede confiabilidade do instrumento, mas sim a concordância entre observadores. Deve-se levar em consideração que o tempo decorrido entre a entrevista e a revisita apresentou mediana de 15 dias. A pergunta escolhida poderia, em algumas ocasiões, gerar um viés, pois o entrevistado poderia confundir todo o processo de entrevista com alguma espécie de aconselhamento.

\section{Discussão}

O conhecimento sobre exercício físico mostrou-se diferente entre homens e mulheres. Também os fatores envolvidos na determinação de tal conhecimento variaram entre os sexos, sendo que apenas escolaridade e nível social apresentaram associações na mesma direção em ambos os sexos. Tais diferenças talvez se expliquem por uma possível diferente visão que homens e mulheres possam ter sobre exercício físico.

Considerou-se satisfatório o nível de informação da população sobre a freqüência mínima necessária para que o exercício físico gere benefícios à saúde, tendo em vista que mais da metade respondeu acertadamente que a partir de três sessões semanais de trinta minutos os ganhos começam a surgir. Cabe destacar que um número maior de sessões poderia gerar benefícios maiores, no entanto esta avaliação visava a identificar o conhecimento apenas em relação à quantidade mínima de sessões. Esta alternativa do questionário seria a mais semelhante quando comparada às recomendações internacionais 10. Esta conclusão é reforçada pelo fato de que menores prevalências foram encontradas nas alternativas mais incorretas, fosse na freqüência semanal ou na duração da sessão.

Com relação ao melhor exercício físico para o emagrecimento com base nas alternativas apresentadas, a maior prevalência foi encontrada para caminhada, concordando com as recomendações baseadas nos princípios da fisiologia, que consideram caminhar um exemplo perfeito de exercício aeróbico - tipo de atividade que mais possivelmente permitiria o consumo de gordura corporal 11,12. Nesta questão surgiu a maior diferença encontrada entre homens e mulheres, uma vez que a quantidade de homens que considera o futebol como o melhor exercício físico para o emagrecimento foi quatro vezes maior.

Muitos ainda vinculam a possibilidade de realizar exercício físico a características como pouca idade e saúde perfeita. Sabe-se hoje que justamente os que mais se beneficiam do movimento com ganhos na qualidade de vida são as pessoas que possuem limitações e/ou idades avançadas 13, no entanto mais de um terço da população ainda considera que fatores como osteoporose, problemas cardíacos e ser idoso são barreiras que impedem a prática de exercícios físicos.

Na análise da percepção, cabe destacar que entre os cinco problemas mais citados pela população como possivelmente alteráveis pelo exercício físico, o estresse apareceu em primeiro lugar, seguido dos problemas circulatórios e logo após apareceram outros problemas considerados da esfera emocional, como depressão e ansiedade, sendo que o quinto problema foi a insônia.

Com exceção dos problemas circulatórios, outros agravos à saúde comumente vinculados ao sedentarismo estavam entre aqueles menos identificados, dentre as opções das doenças para as quais o exercício físico é classicamente considerado como um fator de proteção. Apesar de toda divulgação do exercício físico como protetor contra cardiopatias e outras doenças crônicas, os benefícios mais lembrados pelas 
Regressão linear ajustada entre o conhecimento, sexo, idade, cor da pele, nível social, escolaridade, atividade física e IMC, para toda amostra e estratificada por sexo. Pelotas, Rio Grande do Sul, Brasil, 2002.

\begin{tabular}{|c|c|c|c|c|c|c|}
\hline \multirow[t]{2}{*}{ Variável1 } & \multicolumn{2}{|c|}{ Todos $(n=3.165)$} & \multicolumn{2}{|c|}{ Mulheres $(n=1.759)$} & \multicolumn{2}{|c|}{ Homens $(n=1.344)$} \\
\hline & Coeficiente $\beta$ (IC95\%) & $p$ & Coeficiente $\beta$ (IC95\%) & $p$ & Coeficiente $\beta$ (IC95\%) & $p$ \\
\hline Sexo & & $<0,001$ & & & & \\
\hline Masculino & - & & & & & \\
\hline Feminino & $0,91(0,64$ a 1,17$)$ & & & & & \\
\hline Idade & & $<0,001$ & & $<0,001$ & & 2 \\
\hline $20-29$ & $2,75(2,02$ a 3,47$)$ & & $3,70(2,82$ a 4,57$)$ & & & \\
\hline $30-39$ & $2,95(2,18$ a 3,72$)$ & & $3,91(3,09$ a 4,73$)$ & & & \\
\hline $40-49$ & $2,98(2,20$ a 3,75$)$ & & $3,63(2,68$ a 4,60$)$ & & - & \\
\hline $50-59$ & $2,97(2,13$ a 3,81$)$ & & $3,77(2,80$ a 4,74$)$ & & & \\
\hline $60-69$ & $2,04(1,35$ a 2,73$)$ & & $2,60(1,80$ a 3,41$)$ & & & \\
\hline$\geq 70$ & - & & - & & & \\
\hline Cor da pele & & 0,01 & & 0,008 & & 0,3 \\
\hline Branca & $0,61(-1,09$ a $-0,13)$ & & $0,83(0,22$ a 1,44$)$ & & $0,32(-0,28$ a 0,93$)$ & \\
\hline Negra/Parda & - & & - & & - & \\
\hline Nível social & & $<0,001$ & & $<0,001$ & & $<0,001$ \\
\hline Nível A & $2,10(1,26$ a 2,94$)$ & & $2,22(1,03$ a 3,41$)$ & & $2,30(1,19$ a 3,40$)$ & \\
\hline Nível B & $1,75(1,02$ a 2,47$)$ & & $1,74(0,66$ a 2,83$)$ & & $2,02(1,06$ a 2,98$)$ & \\
\hline Nível C & $1,49(0,75$ a 2,22$)$ & & $1,59(0,52$ a 2,67$)$ & & $1,61(0,65$ a 2,57$)$ & \\
\hline Nível D & $0,74(-0,01$ a 1,49$)$ & & $0,83(-0,20$ a 1,86$)$ & & $0,87(-0,13$ a 1,88$)$ & \\
\hline Nível E & - & & - & & - & \\
\hline Escolaridade & & $<0,001$ & & $<0,001$ & & $<0,001$ \\
\hline 0 & - & & - & & - & \\
\hline $1-4$ & $1,65(0,85$ a 2,45$)$ & & $1,75(0,75$ a 2,75$)$ & & $1,15(0,03$ a 2,27$)$ & \\
\hline $5-8$ & $2,53(1,85$ a 3,20$)$ & & $2,94(2,07$ a 3,81$)$ & & $1,55(0,50$ a 2,60$)$ & \\
\hline $9-11$ & $3,53(2,76$ a 4,31$)$ & & $3,55(2,62$ a 4,49$)$ & & $2,82(1,71$ a 3,94$)$ & \\
\hline$\geq 12$ & $4,52(3,78$ a 5,25$)$ & & $4,38(3,42$ a 5,33$)$ & & $3,76(2,53$ a 4,99$)$ & \\
\hline Situação conjugal & & 2 & & 0,03 & & 2 \\
\hline Com companheiro & - & & $0,39(0,02$ a 0,75$)$ & & - & \\
\hline Sem companheiro & & & - & & & \\
\hline Atividade física & & 0,001 & & 0,001 & & 2 \\
\hline Suficientemente ativo & $0,54(0,23$ a 0,84$)$ & & $0,63(0,27$ a 0,98$)$ & & - & \\
\hline Insuficientemente ativo & - & & - & & & \\
\hline IMC & & 0,002 & & 2 & & 0,03 \\
\hline Desnutrição/Normal $(<25,0)$ & - & & & & - & \\
\hline Sobrepeso $(25,0-29,9)$ & $0,22(-0,1$ a 0,54$)$ & & - & & $0,18(-0,30$ a 0,66$)$ & \\
\hline Obesidade $(\geq 30,0)$ & $0,72(0,29$ a 1,14$)$ & & & & $0,72(0,16$ a 1,29$)$ & \\
\hline
\end{tabular}

Nota: As categorias de base são aquelas com os piores escores.

1 As variáveis: tabagismo (homens), prática de religião e autopercepção de saúde (mulheres) foram levadas

para a análise ajustada, porém como perderam significância não estão mostradas nesta tabela.

2 Valores não significativos na análise bivariada $(p>0,2)$. 
pessoas não são sobre os males puramente físicos, mais facilmente mensurados e associados ao exercício físico. Talvez essa noção devase ao fato que independente de diagnósticos médicos e exames laboratoriais, mesmo pessoas desinformadas conseguem perceber alterações positivas no seu bem-estar advindas do exercício físico. A facilidade de prescrição e a maior adesão aos tratamentos farmacológicos também poderiam reforçar a noção de que problemas como hipertensão e hipercolesterolemia pudessem ser tratados apenas com medicação e dietas, o que poderia levar a população a considerar o exercício físico como menos benéfico no combate a estas morbidades.

As baixas prevalências do reconhecimento de que o exercício físico pode combater o diabetes, além do que aproximadamente mais de um quarto da população não reconhece o exercício físico como protetor contra osteoporose e hipertensão, foram achados considerados negativos no presente estudo.

As diferentes redações das questões que abordavam conceitos de causalidade, prevenção e combate de algumas doenças também podem ter levado os entrevistados a respostas diferenciadas no que diz respeito ao verdadeiro poder do exercício físico sobre os males citados.

Comparativamente às mulheres, os homens recebem menos informação dos médicos e mais de professores, o que poderia ser explicado pela maior freqüência das mulheres em consultórios médicos 14. Por outro lado, os homens de maneira geral, mantêm uma relação mais freqüente com professores e treinadores de esportes coletivos. Contudo, os números extremos são preocupantes, uma vez que quase um quarto dos homens ou um quinto das mulheres referem nunca ter recebido informação sobre os benefícios do exercício físico.

The Writing Group for the Activity Counseling Trial Research Group 15 mostrou que mulheres são mais suscetíveis ao aconselhamento, e que intervenções no sentido de diminuir o sedentarismo possuem um maior impacto sobre elas quando comparadas aos homens.

No presente estudo, as mulheres mesmo possuindo mais conhecimento, não se mostraram mais ativas do que os homens, o que não anula a importância do conhecimento. A maioria dos estudos populacionais utiliza questionários para avaliar os níveis de atividade física, o que para populações é o mais viável. Entretanto, estes inquéritos raramente conseguem medir com fidedignidade satisfatória os níveis de exercício físico. A atividade física por si só, não implica necessariamente ganhos no condicionamento físico como, por exemplo, maior consumo de oxigênio e melhor saúde cardiovascular, que junto a outras condições, levam à menor morbi-mortalidade por qualquer causa.

Para viabilizar a análise do IMC em uma amostra relativamente grande, os dados de peso e altura foram auto-referidos. Esta estratégia não é indicada para pesquisas que procuram analisar a obesidade como foco principal. De acordo com outros estudos sobre o assunto, 16,17 isso poderia alterar pouco a categorização do IMC em algumas parcelas da população, principalmente em mulheres de idade mais avançada ou em situação de obesidade. Esse erro implicaria pequenas superestimativas do sobrepeso e subestimativas da obesidade. Como a informação colhida foi o peso e a altura e a análise do IMC foi feita em categorias, dificilmente estes erros fariam com que um número significativo de pessoas mudassem de categoria na classificação do IMC.

Uma limitação de delineamentos do tipo transversal, que pode ter ocorrido no presente estudo é a causalidade reversa, que pode dificultar a determinação temporal da relação entre causa e efeito. Ou seja, se o conhecimento precedeu a prática de atividade física ou ele é conseqüência desta. Outra limitação particular deste estudo é o fato de que o instrumento foi criado e aplicado pela primeira vez em uma população. Outro aspecto reside na elaboração do escore, que, embora baseado em literatura atual, era formado por valores arbitrários atribuídos pelo criador do questionário.

No presente estudo, os fatores mais envolvidos na determinação do conhecimento foram o nível social e o grau de escolaridade, uma vez que estas variáveis apresentaram forte associação em todos os grupos e com tendência linear. A compreensão desta relação fica clara considerando que o conhecimento sobre exercício físico é parte do conhecimento geral, e que este está vinculado às condições sociais da população.

\section{Conclusões e recomendações}

Mulheres possuem um maior esclarecimento sobre o exercício físico, mesmo assim não apresentaram, neste estudo, maiores níveis de atividade física. Independente de sexo, existe uma forte associação entre este conhecimento e o nível social e a escolaridade.

As diferenças encontradas entre os resultados de homens e mulheres e níveis sociais, bem como diferenças entre grupos etários, devem ser levadas em conta no planejamento de programas abrangentes de incentivo ao exercício físico. 
A criação de locais públicos destinados ao exercício físico, juntamente com programas locais de divulgação sobre atividade física, pode ser uma ferramenta efetiva no combate ao sedentarismo, principalmente se focalizar esforços nestes dois campos - aconselhamento médico a toda população e ações no âmbito escolar para o incentivo e esclarecimento sobre este tema, mais precocemente. Tais estratégias poderiam possibilitar que, após intervenções, fossem feitas reavaliações para determinar o real impacto destas políticas sobre o comportamento da população.

\section{Resumo}

O objetivo deste estudo foi avaliar a percepção e o conhecimento sobre o exercício físico em uma população adulta urbana e estudar a associação entre o nível de conhecimento e características sócio-econômicas, demográficas e comportamentais. O delineamento utilizado foi do tipo transversal, de base populacional, $e$ incluiu 3.182 indivíduos com idade igual ou superior a 20 anos. O questionário desenvolvido para pesquisa gerou um escore de conhecimento ( 0 a 25 pontos). $O$ escore médio na população foi de 17,1 (DP=4,0). Após ajuste, o sexo feminino, a faixa etária entre 30 e 40 anos, elevados níveis social e de escolaridade, mostraram-se fortemente associadas ao desfecho $(p<0,001)$. Também estiveram associados $(p \leq 0,01)$ maiores níveis de atividade física, cor da pele branca e maiores Índices de Massa Corporal (IMC). Os resultados indicam que benefícios específicos do exercício são desconhecidos por parcelas da população. O aconselhamento sobre as vantagens de uma vida ativa não é uma prática comum entre professores.

Exercício Físico; Saúde do Adulto; População Urbana
Futuros estudos que avaliem as questões compreendidas pela relação entre exercício físico e saúde poderão colaborar para a diminuição dos níveis de sedentarismo em qualquer população. Além disso, a utilização de subamostras da população, que permitissem um estudo individual e mais detalhado sobre o condicionamento físico, poderia explicar melhor a relação entre conhecimento e prática de exercício físico.

\section{Colaboradores}

C. L. P. Araújo e D. P. Gigante contribuíram na revisão e correção do artigo, e na elaboração do questionário e do escore analisado. M. R. Domingues participou de todas as etapas da produção do artigo.

\section{Agradecimentos}

Esta pesquisa teve incentivo financeiro da Coordenação de Aperfeiçoamento de Pessoal de Nível Superior. 


\section{Referências}

1. Blair SN, Booth M, Gyarfas I, Iwane H, Marti B, Matsudo V, et al. Development of public policy and physical activity initiatives internationally. Sports Med 1996; 21:157-63.

2. Pate RR, Pratt M, Blair SN, Haskell WL, Macera CA, Bouchard C. Physical activity and public health: a recommendation from the Centers for Disease Control and Prevention and the American College of Sports Medicine. JAMA 1995; 273:402-7.

3. Caspersen CJ, Powell KE, Christenson GM. Physical activity, exercise, and physical fitness: definitions and distinctions for health-related research. Public Health Report 1985; 100:126-31.

4. Vuori I, Paronen O, Oja P. How to develop local physical activity promotion programmes with national support: the Finnish experience. Patient Educ Couns 1998; 33 (1 Suppl):S111-9.

5. Pitanga FJG. Epidemiologia da atividade física, exercício físico e saúde. Salvador: Editora do $\mathrm{Au}-$ tor; 2001.

6. World Health Organization. World Health Day 2002 "Move for Health". Department of Noncommunicable Disease Prevention \& Health Promotion. http:// www.who.int/world-health-day (acessado em 04/Nov/2002).

7. Associação Nacional de Empresas de Pesquisas. Critério de classificação econômica Brasil. http:// www.anep.org.br/m-arquivo.htm (acessado em 07/Fev/2002).

8. World Health Organization. Exercise for health. WHO/FIMS Committee on Physical Activity for Health. Bull World Health Organ 1995; 73:135-6.

9. International Physical Activity Questionnaire. Downloadable questionnaires. http://www.ipaq. ki.se/IPAQ.asp?mnu_sel=DDE\&pg_sel=DDE (acessado em 16/Jan/2002).
10. American College of Sports Medicine. Position stand on the recommended quantity and quality of exercise for developing and maintaining cardiorespiratory and muscular fitness, and flexibility in adults. Med Sci Sports Exerc 1998; 30:975-91.

11. Hawley JA. Fat burning during exercise: can ergogenics change the balance. Phys Sportsmed 1998; http://www.physsportsmed.com/issues/ 1998/09sep/hawley.htm (acessado em 16/Jan/ 2003).

12. Spriet LL. Regulation of skeletal muscle fat oxidation during exercise in humans. Med Sci Sports Exerc 2002; 34:1477-84.

13. Sociedade Brasileira de Medicina do Esporte/Sociedade Brasileira de Geriatria e Gerontologia. Posicionamento oficial da Sociedade Brasileira de Medicina do Esporte e da Sociedade Brasileira de Geriatria e Gerontologia: atividade física e saúde no idoso. Rev Bras Med Esporte 1999; 5:207-11.

14. Dias da Costa JS, Facchini LA. Use of outpatient services in an urban area of Southern Brazil: place and frequency. Rev Saúde Pública 1997; 31:360-9.

15. The Writing Group for the Activity Counseling Trial Research Group. Effects of physical activity counseling in primary care. The activity counseling trial: a randomized controlled trial. JAMA $2001 ; 286: 677-87$.

16. Spencer EA, Appleby PN, Davey GK, Key TJ. Validity of self-reported height and weight in 4808 EPIC-Oxford participants. Public Health Nutr 2002; 5:561-5.

17. Bolton-Smith C, Woodward M, Tunstall-Pedoe H, Morrison C. Accuracy of the estimated prevalence of obesity from self reported height and weight in an adult Scottish population. J Epidemiol Community Health 2000; 54:143-8.

Recebido em 05/Fev/2003

Versão final reapresentada em 24/Jun/2003 Aprovado em 16/Out/2003 